\title{
Cavity quantum electrodynamics in the presence of energy relaxation and pure dephasing: A unified quantum master-equation approach
}

\author{
Ruixue $\mathrm{Xu}$ and YiJing Yan* \\ Open Laboratory of Bond-Selective Chemistry, University of Science and Technology of China, Hefei, China \\ and Department of Chemistry, Hong Kong University of Science and Technology, Kowloon, Hong Kong, China \\ Xin-Qi Li*,† \\ Department of Chemistry, Hong Kong University of Science and Technology, Kowloon, Hong Kong
}

(Received 19 May 2001; published 10 January 2002)

\begin{abstract}
This paper renders two main results. One is a unified quantum master equation, which satisfies the detailedbalance relation and can serve as a convenient starting formulation to study the dissipative dynamics in a variety of systems in quantum optics and solid-state physics. Another is based on the proposed quantum master equation to study cavity quantum electrodynamics in the presence of $T_{1}$ energy relaxation and pure $T_{2}$ dephasing. Demonstrated in detail is also the effect of cavity field-absorber interaction on dissipation, which is neglected in the conventional theory.
\end{abstract}

DOI: $10.1103 /$ PhysRevA.65.023807

PACS number(s): 42.50.Ct, 03.65.Yz, 05.40.-a, 12.20.-m

\section{INTRODUCTION}

The quantization of dissipative systems has a rather long history but still remains as one of the most active research fields due to its ever increasing challenge in new applications. In constructing a quantum dissipation theory, i.e., an equation of motion for reduced system density operator $\rho(t)$, the commonly adopted and maybe also the most successful approach is via the harmonic bath coupling model, in which one explicitly takes into account the interaction between the primary system and a harmonic quantum bath. Since the system-plus bath is a closed system, the standard quantization procedures are readily applicable. A quantum dissipation theory can then be constructed by eliminating the bath variables via various standard techniques [1] such as quantum Langevin equations [2,3], projection operator [3-5], Feynman path integral [6-8], Liouville-space Green function [9-11], dynamical semigroup construction [12-14], and hierarchical or coupled equations of motion [15-20].

We have recently constructed an algebraic form of quantum master equation (QME) that has the advantage of structural simplicity and versatility [21]. The resulting algebraic QME provides also a convenient ground to relate several seemingly different quantum dissipation formulations [21], such as the Bloch-Redfield theory [22-24] and a class of Fokker-Planck equations [7-11,25]. Our previous QME [21] was developed based on a Hermitian decomposition form of system-bath interaction Hamiltonian. This form of systembath coupling is in a sense similar to the Feynman-VernonCaldeira-Leggett model [6-8] that had been widely used in quantum dissipation literature [1].

\footnotetext{
*Corresponding authors. Email address: yyan@ust.hk; email address: xqli@chsg4.ust.hk

${ }^{\dagger}$ Present address: National Laboratory for Superlattices and Microstructures, Institute of Semiconductors, Chinese Academy of Sciences, P. O. Box 912, Beijing 100083, China.
}

This work comprises two contributions. One is the generalization of our previous QME formulation [21] for a nonHermitian decomposition form of system-bath interaction. The resulting unified QME does not only satisfy the detailedbalance relation but also largely preserve the positivity of reduced-density operator in at least the cases of test. It thus is expected to be useful to quantum optics and solid-state physics where the creation and annihilation operators are often the key quantities of study. The unified QME will be presented in Sec. II with the derivation/approximation being given in Appendix A and its Redfield dissipation tensor element analysis in Appendix B.

Another main contribution that will be presented in Sec. III is the study of the dissipative cavity quantum electrodynamics (CQED) via the unified QME. Generally speaking, CQED is of the fundamental physical interest in, for example, the controllable spontaneous emission properties and the strong Rabi oscillations. Moreover, it is also of peculiar value in device applications such as the low-threshold semiconductor lasers [26] and the quantum computing/ information processes [27-33]. In this work, the CQED system will be described by Jaynes-Cummings model, where a two-level absorber is embedded in an optical cavity [34-40]. Physically, the absorber can be either an atom [34-36] or a quantum well exciton [37-40], and interestingly both have similar CQED properties [40]. Most of CQED theories treated energy relaxation due to finite quality in cavity. In Sec. III, we will present studies of $T_{1}$ energy relaxation and pure $T_{2}$ dephasing, due to the finite quality in the cavity or the absorber or both. Together with Appendix C, we will further reveal the effect of cavity-absorber coupling on dissipation that are often neglected by most of the conventional CQED formulations. Finally, we conclude and summarize this paper in Sec. IV.

\section{QUANTUM MASTER EQUATION}

To balance the aforementioned two contributions of this paper, we shall leave the lengthy derivation of the unified 
QME to Appendix A. In this section, we shall present a simplified version of the unified QME that is directly relevant to our study of CQED problems in the next section.

Let us start with the rotating-wave approximation of the Caldeira-Leggett type of system-plus-bath Hamiltonian (denoting $\hbar \equiv 1$ throughout the paper):

$$
H_{T}=H+\sum_{\lambda} \omega_{\lambda}\left(a_{\lambda}-\eta_{\lambda} W\right)^{\dagger}\left(a_{\lambda}-\eta_{\lambda} W\right) \equiv H^{0}+h_{B}+H^{\prime} .
$$

Here

$$
\begin{gathered}
H=H^{0}-W^{\dagger} W \sum_{\lambda} \omega_{\lambda}\left|\eta_{\lambda}\right|^{2}, \\
h_{B}=\sum_{\lambda} \omega_{\lambda} a_{\lambda}^{\dagger} a_{\lambda}, \\
H^{\prime}=-\left(W^{\dagger} f+W f^{\dagger}\right)
\end{gathered}
$$

are the reduced-system Hamiltonian after the renormalization correction [cf. Eq. (A14) and the comments followed], the harmonic-bath Hamiltonian, and the system-bath interaction, respectively. Here, $W$ is an operator in the reducedsystem space and will be specified further in connection with the dissipative CQED problems in Sec. III. In Eq. (2b), $f$ $=\Sigma_{\lambda} \omega_{\lambda} \eta_{\lambda}^{*} a_{\lambda}$ is the generalized Langevin force operator in the stochastic bath space. The bath interaction spectral density function is given by (for $\omega \geqslant 0$ )

$$
J(\omega) \equiv \pi \sum_{\lambda}\left|\eta_{\lambda}\right|^{2} \delta\left(\omega-\omega_{\lambda}\right) .
$$

Mathematically, the definition of $J(\omega)$ is extended to negative frequency domain via $J(-\omega)=-J(\omega)$.

The force-force correlation functions are given by

$$
\widetilde{C}^{(-)}(t) \equiv\left\langle f(t) f^{\dagger}(0)\right\rangle, \quad \widetilde{C}^{(+)}(t) \equiv\left\langle f^{\dagger}(t) f(0)\right\rangle,
$$

with $\langle\cdots\rangle$ denoting an average over the bath canonical ensemble. The bath interaction spectrum is defined as

$$
C^{( \pm)}(\omega)=\int_{-\infty}^{\infty} d t e^{i \omega t} C^{( \pm)}(t) .
$$

It can be shown that $C^{( \pm)}(\omega) \geqslant 0$ and satisfies the detailedbalance relation of [cf. Eq. (A13)]

$$
C^{(+)}(\omega)=e^{\beta \omega} C^{(-)}(-\omega) .
$$

Here, $\beta \equiv \hbar /\left(k_{B} T\right)$. In the present model of system-bath coupling, we can further relate $C^{( \pm)}(\omega)$ to the spectral density function $J(\omega)$ as

$$
C^{( \pm)}(\omega)=\Theta(\mp \omega) \frac{2 J(\omega)}{1-e^{-\beta \omega}} \equiv \Theta(\mp \omega) C(\omega) .
$$

Here, $\Theta(\omega)$ is the Heaviside step function, while $C(\omega)$ defined in the second identity of the above equation is the conventional bath interaction spectrum used, for example, in the Caldeira-Leggett bath model $[1,7]$.

The final QME, up to the second-order cummulant expansion in the system-bath coupling, reads as [cf. Eq. (A15)]

$$
\begin{gathered}
\dot{\rho}(t)-i[H, \rho(t)]-\mathcal{R} \rho(t), \\
\mathcal{R} \rho(t)=\frac{1}{2}\left[W^{\dagger}, W^{(-)} \rho(t)-\rho(t) W^{(+)}\right]+\text {H.c. }
\end{gathered}
$$

with

$$
W^{( \pm)} \equiv C^{( \pm)}( \pm \mathcal{L}) W .
$$

In Eq. $(8 \mathrm{c}), C^{( \pm)}( \pm \mathcal{L})$ is a function of reduced system $\mathrm{Li}-$ ouvillian, $\mathcal{L} \equiv[H, \ldots]$, characterized by the bath interaction spectrum $C^{( \pm)}( \pm \omega)$ [Eq. (5)]. In Appendix B, we examine the Redfield dissipation tensor $\mathcal{R}$ [cf. Eq. (8b)] by using the energy $H$-eigenstate representation to elucidate the relation between the QME [Eq. (8)] and the Redfield theory [24].

We shall hereafter call $W$ a dissipative mode, through which the system interacts with the bath. The unified QME with multiple dissipative modes $\left\{W_{\alpha}\right\}$ is presented in Eq. (A15). It is easy to show that the canonical density operator $\rho_{\mathrm{eq}}=e^{-\beta H} / \operatorname{Tr} e^{-\beta H}$ is a stationary solution to the unified QME [cf. Eq. (A16) and the comment following].

\section{APPLICATION TO CQED SYSTEMS}

\section{A. General description}

In this section, we apply the unified QME [Eq. (8)] to a CQED system [34-36]. To elucidate the fundamental physics in this context, we consider a two-level absorber, which can be either a Rydberg atom or a semiconductor exciton, being coupled resonantly to a single-cavity mode. We adopt the Jaynes-Cummings model [41] in which the reducedsystem Hamiltonian assumes

$$
\begin{aligned}
H & =H_{0}+H_{F A} \equiv H_{F}+H_{A}+H_{F A} \\
& =\omega_{F} \hat{a}^{\dagger} \hat{a}+\omega_{A} \hat{b}^{\dagger} \hat{b}+\omega^{\prime}\left(\hat{a} \hat{b}^{\dagger}+\hat{a}^{\dagger} \hat{d}\right) .
\end{aligned}
$$

Here, $\hat{a}\left(\hat{a}^{\dagger}\right)$ and $\hat{b}\left(\hat{b}^{\dagger}\right)$ are the annihilation (creation) operators of the field and the absorber, respectively. The former is a boson, while the latter is a fermion. They satisfy the following relations: $\quad\left[\hat{a}, \hat{a}^{\dagger}\right]=\left\{\hat{b}, \hat{b}^{\dagger}\right\}=1 \quad$ and $\quad \hat{b}^{2}=[\hat{a}, \hat{b}]$ $=\left[\hat{a}, \hat{b}^{\dagger}\right]=0$, where $[\cdot, \cdot \cdot]$ denotes a commutator and $\{\cdot, \cdot\}$ an anticommutator. In Eq. (9), $\omega_{F}, \omega_{A}$, and $\omega^{\prime}$ are the frequencies of the cavity field, the absorber, and the field-absorber interaction, respectively. Special focus will be on the case of resonant CQED in which $\omega_{F}=\omega_{A}$.

In a real system, either the cavity field or the two-level absorber may be of finite quality due to, for example, imperfection of conductivity or the coupling of the absorber to the continuum of electromagnetic field modes and/or vibrational modes (i.e., phonons). This dissipative defects in the cavity or absorber will be described via the dissipative mode $W$ $=\hat{a}$ or $\hat{b}$, respectively, in the present QME description. Most of the previous CQED formulations considered the $T_{1}$ energy relaxation effect $[35,36]$, via the dissipative mode $W$ 
$=a$ to account for the finite quality of cavity. However, the conventional treatment amounts to the approximation of the associating spectrum conjugation $[\mathrm{Eq} .(8 \mathrm{c})]$ by $W^{( \pm)}$ $\approx C^{( \pm)}\left( \pm \mathcal{L}_{0}\right) W$, where $\mathcal{L}_{0} \equiv \mathcal{L}_{F}+\mathcal{L}_{A}$. The cavity fieldabsorber coupling $\mathcal{L}_{F A}$ or $H_{F A}$ was only included in its coherent dynamics contribution but neglected in its correlation with dissipation $[35,36]$. Obviously, the resulting QME does not lead to the proper steady state of $\rho(\infty)=\rho_{\text {eq }}$. The conventional treatment for the $T_{1}$ energy relaxation via the dissipative mode $W=\hat{a}$ or $\hat{b}$ will be outlined in Appendix C.

In the following two subsections, we shall investigate the dissipative CQED in the presence of $T_{1}$ energy relaxation and pure $T_{2}$ dephasing, due to the finite quality in either cavity or absorber or both. The effect of cavity fieldabsorber coupling $H_{F A}$ on dissipation, which is neglected in the conventional treatment, will also be discussed based on the exact implementation of Eq. (8c) via

$$
W_{u v}^{( \pm)} \equiv \sum_{m n}\langle u \mid n\rangle C^{( \pm)}\left( \pm \omega_{m n}\right) W_{m n}\langle n \mid v\rangle .
$$

Here, $|m\rangle$ and $|n\rangle$ are the eigenstates, with the transition frequency $\omega_{m n}$, of the CQED system [Eq. (9)]; whereas, $|u\rangle$ and $|v\rangle$ will be chosen as the zeroth-order states in the absence of the cavity field-absorber coupling.

Note that the effect of field-absorber coupling on dissipation in CQED was first properly considered by Cresser [42], who constructed a QME via the constants of motion or operators that commutes with Jaynes-Cummings Hamiltonian $H$ [Eq. (9)]. It can be shown that Eq. (8) with the Hamiltonian $H$ of Eq. (9) and $W=a$ assumes the same form of Cresser's QME [42]. Our QME is, however, more general and can be applied to a variety of quantum dissipation systems.

\section{B. CQED in the presence of energy relaxation}

Let us first considers the $T_{1}$ energy relaxation in CQED, due to its finite quality in either the cavity field or the absorber. The dissipative mode $W$ in the unified QME [Eq. (8)] is thus chosen as

$$
W_{F}=\hat{a} \quad \text { or } \quad W_{A}=\hat{b} .
$$

In the above equation and hereafter, we recast the dissipative mode as $W_{\alpha}$, with the added subscript to indicate the source of dissipative imperfection. Similarly, $J(\omega)$ [Eq. (3)], $C^{( \pm)}(\omega)$ [Eq. (7)], and $W^{( \pm)}$[Eq. (8c)] will also be associated with the subscript $\alpha$.

As the cavity photon and the absorber are coupled via the Rabi frequency $\omega^{\prime}$ in Eq. (9), either $W_{F}=\hat{a}$ or $W_{A}=\hat{b}$ will lead to energy relaxation in both the field and the absorber. Their spectrum conjugations $W_{\alpha}^{ \pm}$[Eq. (8c) or (10)] will be numerically evaluated for a given bath interaction spectral density $J_{\alpha}(\omega)$ via Eq. (7). For convenience of comparison, we assume both dissipation modes associating with the same bath interaction spectral density of $J_{\alpha}(\omega)=\eta \omega \exp$ $\left(-\omega / \omega_{c}\right)$. Here, $\omega_{c}$ is the cutoff frequency and $\eta$ indicates the bath interaction strength. In the conventional CQED formu-

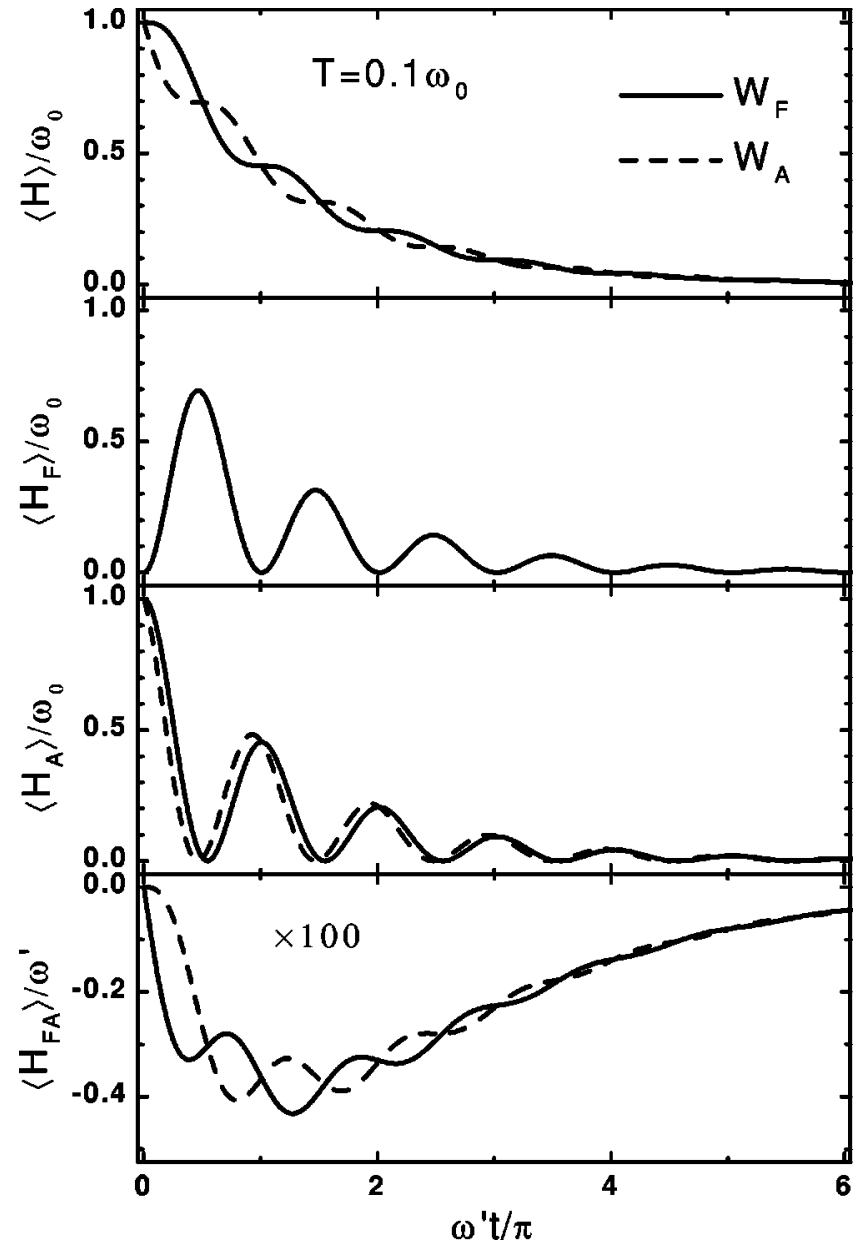

FIG. 1. $T_{1}$ relaxation behaviors of the CQED system. The system is initially in $\rho(0)=\left|0_{F} 1_{A}\right\rangle\left\langle 0_{F} 1_{A}\right|$. Two types of dissipation modes are illustrated, i.e., $W_{F}=\hat{a}$ (solid lines) and $W_{A}=\hat{b}$ (dashed lines). Both dissipation modes are assumed to be associating with the same spectral density of $J(\omega)=\eta \omega \exp \left(-\omega / \omega_{c}\right)$. The relevant parameters are chosen as: $\omega_{F}=\omega_{A}=\omega_{0}, \omega^{\prime}=0.02 \omega_{0}$, and $\omega_{c}$ $=2 \omega_{0} . \eta$ is chosen such that $\gamma_{F}=\gamma_{A}=0.01 \omega_{0}$. The bath temperature is $k_{B} T /\left(\hbar \omega_{0}\right)=0.1$.

lation [Eq. (C1)] one important parameter $\gamma_{\alpha} \equiv 2 J_{\alpha}\left(\omega_{\alpha}\right)$ accounts directly for the dissipative rates (cf. Appendix C).

Figures 1 and 2 demonstrate the effects of energy dissipation resulting individually from $W_{F}=\hat{a}$ and $W_{A}=\hat{b}$ on different energy components of a resonant CQED system, in which $\omega_{F}=\omega_{A}=\omega_{0}$, and $\omega^{\prime}=0.02 \omega_{0}$. The system is assumed to be initially in $\rho(0)=\left|0_{F} 1_{A}\right\rangle\left\langle 0_{F} 1_{A}\right|$, with the field being in the ground state and the absorber in the excited state. Note that in the absence of dissipation $\left\langle H_{F}\right\rangle$ or $\left\langle H_{A}\right\rangle$ would oscillate as $\cos ^{2}\left(\omega^{\prime} t\right)$. The time axis is thus represented in $\omega^{\prime} t / \pi$. For the spectral density $J_{\alpha}(\omega)$ the cutoff frequency is set as $\omega_{c}=2 \omega_{0}$, while the parameter $\eta$ is chosen such that $\gamma_{F}=\gamma_{A}=2 J_{\alpha}\left(\omega_{0}\right)=0.01 \omega_{0}$, which amounts to the quality factor $Q=\omega_{0} / \gamma_{\alpha}=100$ used in the conventional CQED theory. The bath temperature is $k_{B} T /\left(\hbar \omega_{0}\right)$ $=0.1$ for Fig. 1 and 1.0 for Fig. 2. It is obviously seen that the damping is faster for higher temperature. We also find that in the present case of study, the calculated time- 


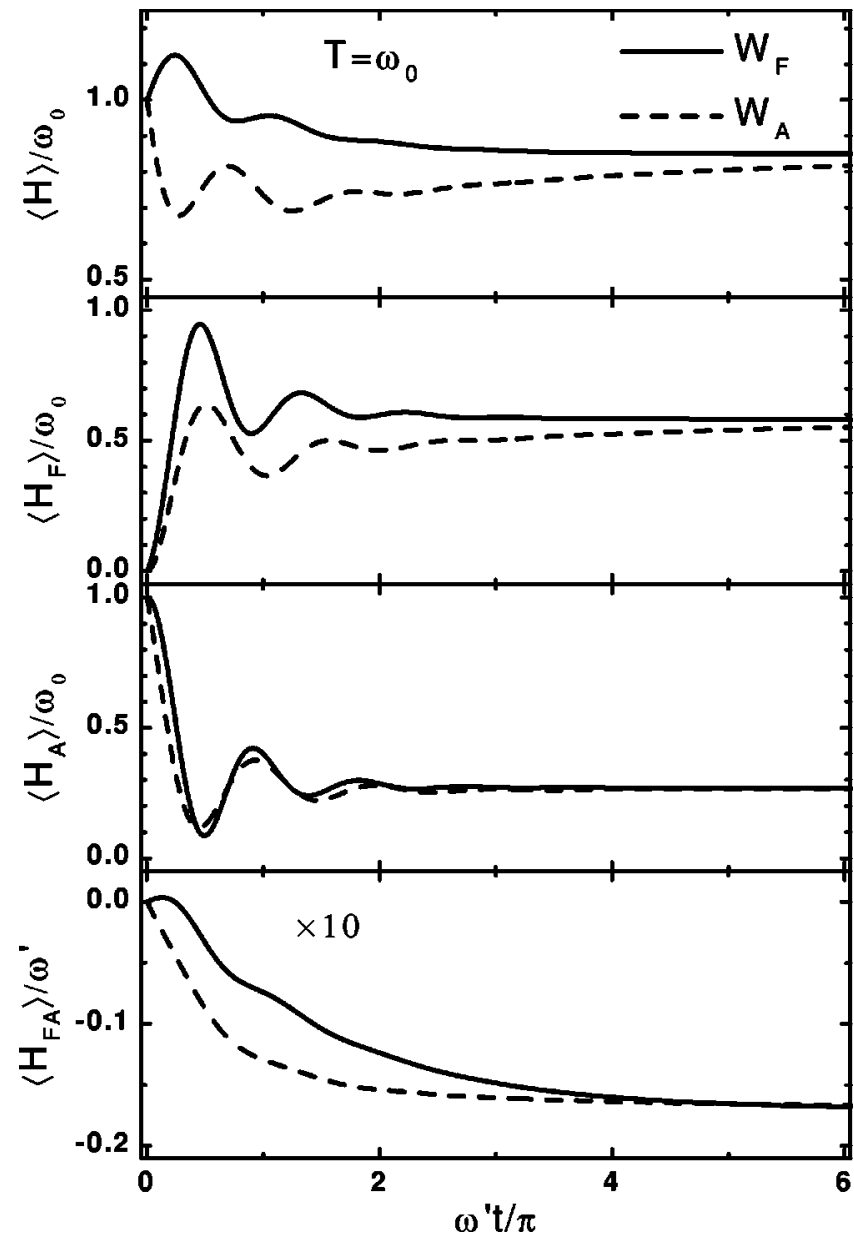

FIG. 2. $T_{1}$ relaxation behaviors of the CQED system for the bath temperature $k_{B} T /\left(\hbar \omega_{0}\right)=1.0$. The other conditions or parameters are chosen the same as Fig. 1.

dependent values of $\langle H\rangle,\left\langle H_{F}\right\rangle$, and $\left\langle H_{A}\right\rangle$ are well close to those obtained via the conventional zeroth-order evolution [cf. Appendix $\mathrm{C}$ where $W^{( \pm)} \approx C^{( \pm)}( \pm \mathcal{L}) W$ is adopted]. As described at the end of Appendix $\mathrm{C},\left\langle H_{F A}\right\rangle$ would remain zero in the conventional treatment. Therefore, the resulting nonzero results of $\left\langle H_{F A}\right\rangle$ shown in the bottom panels of Fig. 1 and Fig. 2 indicate the effect of field-absorber coupling $H_{F A}$ on dissipation, which is neglected in the conventional CQED formalism [35,36]. For Fig. 1 in the case of a rather low temperature, most of the system energy is lost to the bath because of dissipation. Interestingly, the damping of the cavity field $\left\langle H_{F}\right\rangle$ are almost indistinguishable between these two sources of $T_{1}$ relaxation in this case.

\section{CQED in the presence of pure dephasing}

We now turn to the effect of pure dephasing induced via the field-absorber coupling fluctuations. This type of dissipation, which is as realistic as the $T_{1}$ relaxation considered earlier is, however, largely ignored in the conventional CQED theory. In the present unified QME, the field-absorber coupling fluctuation-induced dissipation can be described via the following dissipation mode,

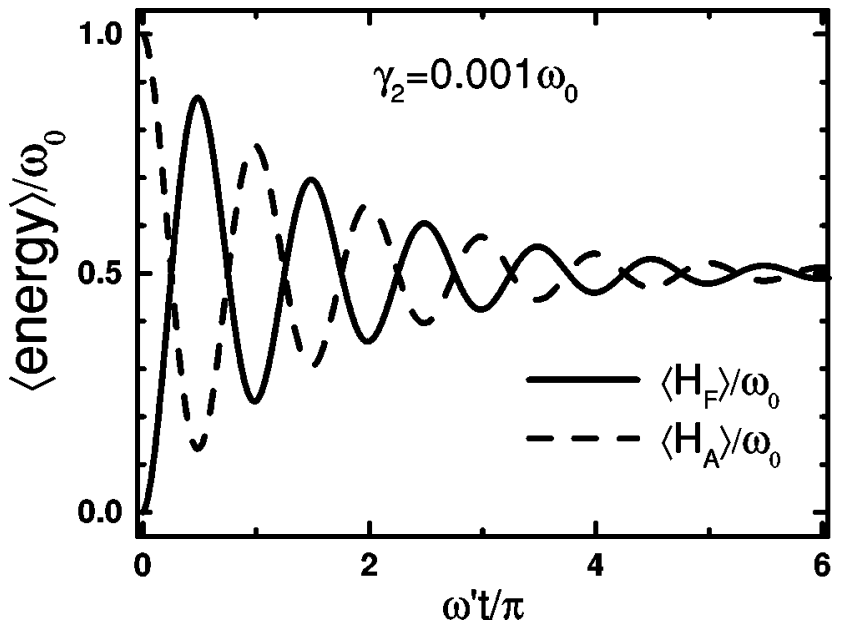

FIG. 3. Demonstration of the pure dephasing of CQED system via the dissipation mode $W_{2}=\hat{a} \hat{b}^{\dagger}+\hat{a}^{\dagger} \hat{b}$. The system parameters and initial state are the same as Fig. 1. The pure dephasing rate is chosen as $\gamma_{2}=0.001 \omega_{0}$.

$$
W_{2}=H_{F A} / \omega^{\prime}=\hat{a} \hat{b}^{\dagger}+\hat{a}^{\dagger} \hat{b} .
$$

This dissipation mode is Hermitian and satisfies $\left[H, W_{2}\right]$ $=\left(\omega_{A}-\omega_{F}\right)\left(\hat{a} \hat{b}^{\dagger}-\hat{a}^{\dagger} \hat{b}\right)$. In the case of $\omega_{F}=\omega_{A}=\omega_{0}$, we have $\left[H, W_{2}\right]=0$. Therefore, in the resonance condition the dissipative mode $W_{2}$ [Eq. (12)] causes no energy loss but only pure $T_{2}$ dephasing [21]. The corresponding dissipation term in QME [Eq. (8)] is given by [21]

$$
\mathcal{R} \rho=\gamma_{2}\left(\hat{a} \hat{b}^{\dagger}+\hat{a}^{\dagger} \hat{b},\left[\hat{a} \hat{b}^{\dagger}+\hat{a}^{\dagger} \hat{b}, \rho\right]\right) .
$$

Here, $\gamma_{2} \equiv C_{2}(0) / 2$ is the pure dephasing rate. Obviously, the dissipation term in Eq. (13) is of the Lindblad's semigroup form [12-14].

Figure 3 shows the evolution of the CQED energies in the presence of only this type of dissipation, with $\gamma_{2}$ $=0.001 \omega_{0}$. The initial condition and the coherent frequencies of the CQED are chosen as the same of those in Fig. 1. We find that the absorber relaxes from the initial upper state to a mixture of the two-level states; meanwhile, its energy is partially transfered to the cavity photon that evolves also into a mixed state. However, as we pointed out earlier, the total energy (not shown) of the system is conserved.

\section{CONCLUDING REMARKS}

To summarize, we have proposed a unified QME [Eq. (8) or Eq. (A15)] that may serve as a convenient starting point to study a wide variety of dissipation phenomena, especially in quantum optics and solid-state physics. Demonstrated is also the Feynman-Veron-Caldeira-Leggett bath interaction model but used in the context of rotation-wave approximation [cf. Eq. (1)] for the unified QME. We have further studied the simplest CQED system [Eq. (9)] in the presence of $T_{1}$ energy relaxation and pure $T_{2}$ dephasing via the unified QME formulation. Besides demonstrating that all the conventional CQED formulations can be easily obtained in the unified QME framework, we also reveal some dissipative effects 
that are not accounted for by the conventional theory.

\section{ACKNOWLEDGMENTS}

Support from the Research Grants Council of the Hong Kong Government and the National Natural Science Foundation of China is gratefully acknowledged.

\section{APPENDIX A: DERIVATION OF THE UNIFIED QUANTUM MASTER EQUATION}

\section{General formulation of quantum dissipation}

For the general interest in dissipative dynamics, we shall in this appendix construct a unified QME and discuss in detail the approximations involved. We shall show that Eq. (8) is only a simplified version of the QME to be developed in this appendix.

Without losing the generality, let us start with a stochastic description in which the total Hamiltonian of system plus bath assumes the form [cf. the second identity of Eq. (1)]

$$
H_{T}(t)=H^{0}+H^{\prime}(t) .
$$

Here, $H^{0}$ is the reduced-system Hamiltonian before the renormalization correction [cf. Eq. (2a)], while $H^{\prime}(t)$ $\equiv e^{i h_{B} t} H^{\prime} e^{-i h_{B} t}$ is the stochastic system-bath interaction governing dissipation. The latter is assumed to be weak and of statistical zero mean, $\left\langle H^{\prime}(t)\right\rangle=0$, for its average over the initial bath thermal ensemble at temperature $T$.

By using the second-order cummulant expansion, one can arrive at the following Liouville-von Neumann equation [3-5,19-21]:

$$
\begin{gathered}
\dot{\rho}(t)=-i \mathcal{L}^{0} \rho(t)-\mathcal{R} \rho(t), \\
\mathcal{R}=\int_{-\infty}^{t} d_{\tau}\left\langle\mathcal{L}^{\prime}(t) \mathcal{G}^{0}(t, \tau) \mathcal{L}^{\prime}(\tau) \mathcal{G}^{0 \dagger}(t, \tau)\right\rangle .
\end{gathered}
$$

Here,

$$
\begin{gathered}
\mathcal{L}^{0} \equiv\left[H^{0}, \ldots\right], \quad \mathcal{L}^{\prime}(t) \equiv\left[H^{\prime}(t), \ldots\right] \\
\mathcal{G}^{0}(t, \tau)=e^{-\mathcal{L}^{0}(t-\tau)}
\end{gathered}
$$

In deriving Eq. (A2), we have set the initial time $t_{0}$ as $-\infty$. By doing that, the initial factorization approximation may be avoided $[18,20]$.

In the presence of time-dependent external field, $H^{0}$ or $\mathcal{L}^{0}$ will explicitly depend on time and Eq. (A3b) should be replaced by a time-ordered exponential. In this case, the superoperator $\mathcal{R}$ [Eq. (A2b)] will describe the correlated drivingdissipation dynamics, which under certain conditions may lead to a dramatic cooperative phenomena, i.e., quantum stochastic resonance [43-45]. We shall not consider this situation here.

The evaluation of $\mathcal{R}$ via Eq. (A2b) involves numerically tedious tensor algebra. Furthermore, the quantity inside the bath ensemble average, $\langle\cdots\rangle$, contains also reduced-system variables in Liouville space, which is often too complicated for Eq. (A2b) to be practically useful. The further formulation development should be made with a certain form of the system-bath coupling.

In this paper, we shall adopt the following decomposition form for the system-bath interaction [cf. Eq. (2c)]:

$$
H^{\prime}(t)=-\sum_{\alpha}\left[W_{\alpha}^{\dagger} f_{\alpha}(t)+W_{\alpha} f_{\alpha}^{\dagger}(t)\right]
$$

Here, $W_{\alpha}\left(f_{\alpha}\right)$ is an operator in the reduced-system (bath) space. The special case in which both $W_{\alpha}$ and $f_{\alpha}$ are Hermitian was treated in our previous work [21].

In general, we need to consider the following four types of stationary force-force correlation functions:

$$
\begin{aligned}
& \widetilde{C}_{\alpha \alpha^{\prime}}^{(-)}(t) \equiv\left\langle f_{\alpha}(t) f_{\alpha^{\prime}}^{\dagger}(0)\right\rangle, \\
& \widetilde{C}_{\alpha \alpha^{\prime}}^{(+)}(t) \equiv\left\langle f_{\alpha}^{\dagger}(t) f_{\alpha^{\prime}}(0)\right\rangle ;
\end{aligned}
$$

and

$$
\begin{aligned}
& \widetilde{C}_{\alpha \alpha^{\prime}}^{(-)^{\prime}}(t) \equiv\left\langle f_{\alpha}^{\dagger}(t) f_{\alpha^{\prime}}^{\dagger}(0)\right\rangle, \\
& \widetilde{C}_{\alpha \alpha^{\prime}}^{(+)^{\prime}}(t) \equiv\left\langle f_{\alpha}(t) f_{\alpha^{\prime}}(0)\right\rangle .
\end{aligned}
$$

They satisfy

$$
\begin{gathered}
{\left[\widetilde{C}_{\alpha \alpha^{\prime}}^{( \pm)}(t)\right]^{*}=\widetilde{C}_{\alpha^{\prime} \alpha}^{( \pm)}(-t),} \\
{\left[\widetilde{C}_{\alpha \alpha^{\prime}}^{( \pm)^{\prime}}(t)\right]^{*}=\widetilde{C}_{\alpha^{\prime} \alpha}^{(\mp)^{\prime}}(-t) .}
\end{gathered}
$$

By using Eqs. (A4)-(A6), together with the same elementary Liouville-space algebra [21], we can recast Eq. (A2b) as

$$
\begin{aligned}
\mathcal{R} \rho(t)= & \frac{1}{2} \sum_{\alpha}\left\{\left[W_{\alpha}^{\dagger}, W_{\alpha}^{(-)} \rho(t)-\rho(t) W_{\alpha}^{(+)}\right]\right. \\
& \left.+\left[W_{\alpha}, W_{\alpha}^{(-)^{\prime}} \rho(t)-\rho(t) W_{\alpha}^{(+)^{\prime}}\right]\right\}+ \text { H.c. }
\end{aligned}
$$

Here,

$$
\begin{gathered}
W_{\alpha}^{(-)} \equiv 2 \sum_{\alpha^{\prime}} \int_{0}^{\infty} d \tau \widetilde{C}_{\alpha \alpha^{\prime}}^{(-)}(\tau) e^{-i \mathcal{L}^{0} \tau} W_{\alpha^{\prime}}, \\
W_{\alpha}^{(+)} \equiv 2 \sum_{\alpha^{\prime}} \int_{0}^{\infty} d \tau\left[\widetilde{C}_{\alpha \alpha^{\prime}}^{(+)}(\tau)\right]^{*} e^{-i \mathcal{L}^{0} \tau} W_{\alpha^{\prime}},
\end{gathered}
$$

and $W_{\alpha}^{( \pm)^{\prime}}$ defined similarly by replacing $C_{\alpha \alpha^{\prime}}^{( \pm)}$with $\widetilde{C}_{\alpha \alpha^{\prime}}^{( \pm)^{\prime}}$. Equation (A8) together with Eqs. (A2a) and (A9) constitute the formal quantum dissipation theory, in which the systembath interaction is taken into account up to the second-order cummulant expansion.

Note that the dissipation superoperator $\mathcal{R}$ in Eq. (A8) does not depend on time as long as $\mathcal{L}^{0}$ is time independent, no matter what the time scales are in the bath correlation functions of Eqs. (A5) and (A6). A quantum dissipation theory with a time-independent $\mathcal{R}$ is traditionally considered as Markovian. Equations (A8) and (A9) thus attribute this 
Markovian nature to the neglect of direct cooperation between time-dependent coherent driving and dissipation [21].

\section{Unified QME: Construction and approximations}

We are now in a position to construct the unified QME, a simplified version of which was adopted in Sec. II and applied to the CQED problems in Sec. III. The unified QME will be constructed with two more approximations to be detailed as follows.

One is the rotating-wave approximation (RWA) in which we set [cf. Eq. (A6)]

$$
\widetilde{C}_{\alpha \alpha^{\prime}}^{( \pm)^{\prime}}(t)=0 .
$$

This approximation may be justifiable if the generalized Langevin-force operator $f_{\alpha}$ is chosen to be the annihilation operator of bath mode. Actually, for the harmonic bath model considered in Sec. II the above RWA becomes exact. The RWA [Eq. (A10)] amounts to [cf. Eq. (A9) with $C_{\alpha \alpha^{\prime}}^{( \pm)}$replaced by $\left.C_{\alpha \alpha^{\prime}}^{( \pm)^{\prime}}\right]$

$$
W_{\alpha}^{( \pm)^{\prime}}=0 .
$$
[21]

Another is the so-called Redfield approximation in which

$$
2 \int_{0}^{\infty} d \tau \widetilde{C}_{\alpha \alpha^{\prime}}^{( \pm)}(t) e^{i \omega t} \approx \int_{-\infty}^{\infty} d \tau \widetilde{C}_{\alpha \alpha^{\prime}}^{( \pm)}(\tau) e^{i \omega \tau} \equiv C_{\alpha \alpha^{\prime}}^{( \pm)}(\omega) .
$$

The bath interaction spectra satisfy [cf. Eq. (6)]

$$
\left[C_{\alpha \alpha^{\prime}}^{( \pm)}(\omega)\right]^{*}=C_{\alpha^{\prime} \alpha}^{( \pm)}(\omega)=e^{\beta \omega} C_{\alpha \alpha^{\prime}}^{(\mp)}(-\omega) .
$$

The second identity above is the spectral detailed-balance relation.

To proceed, let us comment on the physical implication of the Redfield approximation, Eq. (A12). It can be shown that $C_{\alpha \alpha}^{( \pm)}(\omega)$ is real and non-negative [cf. the first identity of Eq. (A13) with $\alpha=\alpha^{\prime}$ ]. Thus, the Redfield approximation in this case amounts to the neglect of bath dispersion, i.e., the imaginary part of the first quantity in Eq. (A12). The bath dispersion affects not only the dissipative dynamics but also the thermal equilibrium state $\rho_{\text {eq }}$ being approached by the reduced-density operator $[1,21]$. In fact, if we cast $\rho_{\text {eq }}$ $=e^{-\beta H_{\text {eff }} / \operatorname{Tr}} e^{-\beta H_{\text {eff }}}$, the effective reduced-system Hamiltonian will be temperature-dependent $[1,20]$. To a certain extent [20], this effective Hamiltonian may be well approximated by the Caldeira-Leggett's form of renormalized reduced-system Hamiltonian [cf. Eq. (2a)],

$$
H=H^{0}-H_{\text {ren }} .
$$

In other words, $\mathcal{L}^{0} \equiv\left[H^{0}, \cdot\right]$ in both Eqs. (A2) and (A9) should be replaced by $\mathcal{L} \equiv[H, \cdot]$, in order to keep consistency once the Redfield approximation [Eq. (A12)] is adopted [20]. Further justification will be made to the end of this appendix, where we will show that $\rho_{\mathrm{eq}}=e^{-\beta H} / \operatorname{Tr} e^{-\beta H}$ does constitute a stationary solution to the resulting QME.
The unified QME, which is obtained by invoking the aforementioned two approximations, can now be summarized as

$$
\begin{gathered}
\dot{\rho}(t)=-i[H, \rho(t)]-\sum_{\alpha} \mathcal{R}_{\alpha} \rho(t), \\
\mathcal{R}_{\alpha}=\frac{1}{2}\left[W_{\alpha}^{\dagger}, W_{\alpha}^{(-)} \rho(t)-\rho(t) W_{\alpha}^{(+)}\right]+\text {H.c. }
\end{gathered}
$$

with

$$
\begin{gathered}
W_{\alpha}^{(-)}=\sum_{\alpha^{\prime}} C_{\alpha \alpha^{\prime}}^{(-)}(-\mathcal{L}) W_{\alpha^{\prime}}, \\
W_{\alpha}^{(+)}=\sum_{\alpha^{\prime}} C_{\alpha^{\prime} \alpha}^{(+)}(\mathcal{L}) W_{\alpha^{\prime}} .
\end{gathered}
$$

Here, $C_{\alpha \alpha^{\prime}}^{( \pm \pm}( \pm \mathcal{L})$ is a function of reduced-system Liouvillian operator, defined by the bath interaction spectrum $C_{\alpha \alpha^{\prime}}^{( \pm)}(\omega)$ [Eq. (A12)]. The detailed-balance relation of Eq. (A15) can be easily verified by using the second identity of Eq. (A13), which leads to

$$
W_{\alpha}^{(+)}=e^{\beta \mathcal{L}} W_{\alpha}^{(-)} \equiv e^{\beta H} W_{\alpha}^{(-)} e^{-\beta H} .
$$

Thus, $\rho_{\mathrm{eq}}=e^{-\beta H} / \operatorname{Tr} e^{-\beta H}$ does constitute a stationary solution to Eq. (A15). Obviously, Eq. (A15) reduces to Eq. (8) if there is only a single dissipative mode.

\section{APPENDIX B: REDFIELD TENSOR ELEMENTS}

To elucidate the relation between the QME [Eq. (8)] and the Redfield theory [24], we examine the involvement of Redfield dissipation tensor $\mathcal{R}$ [cf. Eq. (8b)] by using the $H$-eigenstate $\{|a\rangle\}$ representation. Note the indexes $a$ and $b$ used in this appendix denote the eigenstate-representation and should not be confused with the annihilation operators $\hat{a}$ and $\hat{b}$ in Sec. III. For simplicity, we consider a single dissipative-mode contribution and neglect its subindex $\alpha$ or $\alpha^{\prime}$.

The explicit expression for the dissipation tensor $\mathcal{R}$ elements can be easily carried out as

$$
\begin{gathered}
\mathcal{R}_{a b, a^{\prime} b^{\prime}}=\frac{1}{2}\left(\mathcal{K}_{a b, a^{\prime} b^{\prime}}+\mathcal{K}_{b a, b^{\prime} a^{\prime}}^{*}\right), \\
\mathcal{K}_{a b, a^{\prime} b^{\prime}}=\delta_{b^{\prime} b}\left[W^{\dagger} W^{(-)}+W W^{(+) \dagger}\right]_{a a^{\prime}} \\
-\left[W_{b b^{\prime}}^{*} W_{\alpha \alpha^{\prime}}^{(-)}+W_{b^{\prime} b} W_{a^{\prime} a}^{(+) *}\right] .
\end{gathered}
$$

It is easy to show that $R_{a b, a^{\prime} b^{\prime}}=R_{b a, b^{\prime} a^{\prime}}^{*}$, and $\Sigma_{a} \mathcal{K}_{a a, a^{\prime} b^{\prime}}$ $=0$. The latter implies $\Sigma_{a} \mathcal{R}_{a a, a^{\prime} b^{\prime}}=0$. This is matter conservation, which is, however, an obvious property in the commutator form of QME, Eq. (8) or (A15). Noting that $W_{a b}^{( \pm)}$ $=C^{( \pm)}\left( \pm \omega_{a b}\right) W_{a b}$ [cf. Eq. (8c)], the two square-bracket terms in Eq. (B1b) can be explicitly expanded as follows: 


$$
\begin{aligned}
& {\left[W^{\dagger} W^{(-)}+W W^{(+) \dagger}\right]_{a a^{\prime}}} \\
& \quad=\sum_{c}\left[W_{c a}^{*} W_{c a^{\prime}} C^{(-)}\left(\omega_{a^{\prime} c}\right)+W_{a c} W_{a^{\prime} c}^{*} C^{(+)}\left(\omega_{a^{\prime} c}\right)\right],
\end{aligned}
$$

and

$$
\begin{aligned}
& W_{b b^{\prime}}^{*}, W_{a a^{\prime}}^{(-)}+W_{b^{\prime} b} W_{a^{\prime} a}^{(+) *} \\
& \quad=W_{b b^{\prime}}^{*} W_{a a^{\prime}} C^{(-)}\left(\omega_{a^{\prime} a}\right)+W_{b^{\prime} b} W_{a^{\prime} c}^{*} C^{(+)}\left(\omega_{a^{\prime} a}\right) .
\end{aligned}
$$

Each individual Redfield relaxation element $\mathcal{R}_{a b, a^{\prime} b^{\prime}}$ in Eq. (B1) is of clearly physical meaning [24]. For example, $-\mathcal{R}_{b b, a a}$; with $n \neq b$, amounts to the transfer of occupation probability from $|a\rangle$ to $|b\rangle$. By using Eqs. (B1)-(B3), we have

$$
-\mathcal{R}_{b b, a a}=\left|W_{b a}\right|^{2} C^{(-)}\left(\omega_{a b}\right)+\left|W_{a b}\right|^{2} C^{(+)}\left(\omega_{a b}\right) .
$$

The effective $T_{1}$ relaxation rate of level $|a\rangle$ can be defined as

$$
\Gamma_{a}=-\sum_{b \neq a} \mathcal{R}_{b b, a a}
$$

By using the spectroscopic detailed-balance relation [cf. Eq. (A13)], Eq. (B4) leads also to

$$
\mathcal{R}_{a a, b b}=e^{-\beta \omega_{a b}} \mathcal{R}_{b b, a a} .
$$

This is the detailed-balance relation. Nevertheless, this property of the unified QME [Eq. (8) or (A15)] can be proved more rigorously and directly with the operator algebra as in Eq. (A16).

Now consider another type of secular tensor elements, $\mathcal{R}_{a b, a b}=\left(\mathcal{K}_{a b, a b}+\mathcal{K}_{b a, b a}^{*}\right) / 2$, which describes the dephasing between two states $(a \neq b)$. By using Eqs. (B1)-(B5), we obtain [denoting $S(0) \equiv C^{(+)}(0)=C^{(-)}(0)$ ]

$$
\begin{aligned}
\mathcal{K}_{a b, a b}= & {\left[W^{\dagger} W^{(-)}+W W^{(+) \dagger}\right]_{a a}-\left[W_{b b}^{*} W_{a a}^{(-)}+W_{b b} W_{a a}^{(+)}\right] } \\
= & \sum_{c}\left[\left|W_{c a}\right|^{2} C^{(-)}\left(\omega_{a c}\right)+\left|W_{a c}\right|^{2} C^{(+)}\left(\omega_{a c}\right)\right] \\
& -S(0)\left[W_{a a} W_{b b}^{*}+W_{a a}^{*} W_{b b}\right] \\
= & \Gamma_{a}+2 S(0)\left[\left|W_{a a}\right|^{2}-\operatorname{Re}\left(W_{a a} W_{b b}^{*}\right)\right] .
\end{aligned}
$$

It leads to [cf. Eq. (B1a)]

$$
\mathcal{R}_{a b, a b}=\frac{1}{2}\left(\Gamma_{a}+\Gamma_{b}\right)+S(0)\left|W_{a a}-W_{b b}\right|^{2} .
$$

Here, the first term corresponds to the $T_{1}$-induced dephasing, while the second term is the pure $T_{2}$-dephasing rate.

\section{APPENDIX C: SOME COMMENTS ON THE CONVENTIONAL CQED FORMALISM}

It is well known that the conventional CQED theory, which considers the dissipative imperfection in cavity but neglects the direct effect of the cavity field-absorber coupling $\mathcal{L}_{F A}$ or $H_{F A}$ on dissipation, does not satisfy the detailed-balance relation. In relation to the present unified QME [Eq. (8)], the conventional one amounts to having the approximation of $W^{( \pm)} \approx C^{( \pm)}\left( \pm \mathcal{L}_{0}\right) W$, where $\mathcal{L}_{0}$ is the Liouvillian for the uncorrelated cavity-absorber system. In this appendix, we shall discuss some other consequences that might be used as experimental verifications of the breakdown of the conventional CQED formulation.

Before proceeding, let us first consider the effect of $H_{F A}$ on dissipation in terms of perturbation expansions (not given explicitly here). The obtained leading correction term for $W^{( \pm)}$is proportional to $\omega^{\prime} \Delta^{ \pm}$; with $\Delta^{ \pm} \equiv\left[C\left( \pm \omega_{F}\right)-C\right.$ $\left.\left( \pm \omega_{A}\right)\right] /\left(\omega_{F}-\omega_{A}\right)$. In the resonant case, $\omega_{0}=\omega_{F}=\omega_{A}$ and $\Delta^{ \pm}=C^{\prime}\left( \pm \omega_{0}\right)$ or the spectrum derivative. Thus, the conventional CQED theory is expected to be valid when the bath interaction spectrum is broad such that $\mid \omega^{\prime} C^{\prime}\left( \pm \omega_{0}\right) / C$ $\left( \pm \omega_{0}\right) \mid \ll 1$.

In the following, we will show that $\left\langle H_{F A}\right\rangle$ [cf. Eq. (C3)], if it is measurable, can be used as an indicator for the breakdown of the conventional CQED formulation. Let us consider the $T_{1}$ energy relaxation via the dissipative mode $W_{F}$ $=\hat{a}$ or $W_{A}=\hat{b}$. By using Eq. (7), together with some elementary algebra of spin and boson systems, we obtain $C^{( \pm)}$ $\left( \pm \mathcal{L}_{0}\right) W_{\alpha}=C\left(\mp \omega_{\alpha}\right) W_{\alpha}$; for $\alpha=A$ or $F$, where $C(\omega)$ defined in the second identity of Eq. (7) is the commonly used bath interaction spectrum [1,7]. We have $C\left(\omega_{\alpha}\right)=\gamma\left(n_{\alpha}\right.$ $+1)$ and $C\left(-\omega_{\alpha}\right)=\gamma n_{\alpha}$, where $\gamma \equiv 2 J\left(\omega_{\alpha}\right)$ and $n_{\alpha}$ $\equiv\left(e^{\beta \omega_{\alpha}-1}\right)^{-1}$. Let us denote the dissipation superoperator in the conventional CQED formulation as $\mathcal{R}^{(0)}$ for its zerothorder in $H_{F A}$. For $W_{F}=\hat{a}$ or $W_{A}=\hat{b}$, we can obtain

$$
\begin{aligned}
\mathcal{R}_{F}^{(0)} \rho= & \gamma_{F}\left(n_{F}+1\right)\left(\left\{\hat{a}^{\dagger} \hat{a}, \rho\right\} / 2-\hat{a} \rho \hat{a}^{\dagger}\right) \\
& +\gamma_{F} n_{F}\left(\left\{\hat{a} \hat{a}^{\dagger}, \rho\right\} / 2-\hat{a}^{\dagger} \rho \hat{a}\right), \\
\mathcal{R}_{A}^{(0)} \rho= & \gamma_{A}\left(n_{A}+1\right)\left(\left\{\hat{b}^{\dagger} \hat{b}, \rho\right\} / 2-\hat{b} \rho \hat{b}^{\dagger}\right) \\
& +\gamma_{A} n_{A}\left(\left\{\hat{b} \hat{b}^{\dagger}, \rho\right\} / 2-\hat{b}^{\dagger} \rho \hat{b}\right),
\end{aligned}
$$

and the conventional CQED formulation amounts to

$$
\dot{\rho}=-i[H, \rho]-\mathcal{R}_{\alpha}^{(0)} \rho .
$$

Obviously, both $\mathcal{R}_{F}^{(0)}$ and $\mathcal{R}_{A}^{(0)}$ are of the Lindblad's semigroup from $[12,14]$. Their physical interpretations are as follows. The first (second) terms in $\mathcal{R}_{F}^{(0)}$ and $\mathcal{R}_{A}^{(0)}$ describe the energy loss (gain) in the cavity field and the absorber, respectively, to (from) the bath. Thus, in the complete absence of field-absorber coupling, the net energy relaxation rate in the cavity field is $\gamma_{F}$ and that in the absorber is $\gamma_{A}$.

Let us now have a look at the consequence of the above zeroth-order QME on the expectation value of $H_{F A}$; i.e.,

$$
\left\langle H_{F A}\right\rangle \equiv \operatorname{Tr}\left[H_{F A} \rho(t)\right] .
$$

Equation (C2) that describes the time evolution of reduceddensity operator can be considered as the Schrödinger prescription. Its Heisenberg prescription for the time evolution 
of an arbitrary reduced system dynamic variable $\hat{O}$ can be readily obtained. In terms of the expectation value, we have

$$
d\langle\hat{O}\rangle / d t=-i\langle[\hat{O}, H]\rangle-\left\langle\hat{O} \mathcal{R}_{\alpha}^{(0)}\right\rangle .
$$

Here, $\hat{O} \mathcal{R}_{\alpha}^{(0)}$ is given, consistent with Eq. (C1a) for $\alpha=F$, as

$$
\begin{aligned}
\hat{O} \mathcal{R}_{F}^{(0)}= & \gamma_{\alpha}\left(n_{\alpha}+1\right)\left(\left\{\hat{O}, \hat{a}^{\dagger} \hat{a}\right\} / 2-\hat{a}^{\dagger} \hat{O} \hat{a}\right) \\
& +\gamma_{\alpha} n_{\alpha}\left(\left\{\hat{O}, \hat{a} \hat{a}^{\dagger}\right\} / 2-\hat{a} \hat{O} \hat{a}^{\dagger}\right) .
\end{aligned}
$$

The expression for $\hat{O} \mathcal{R}_{A}^{(0)}$ can be obtained by replacing $\hat{a}$ with $\hat{b}$ in the above equation.

The above formulation is now applied to $\hat{O}=H_{F A}$ $=\omega^{\prime}\left(\hat{a} \hat{b}^{\dagger}+\hat{a}^{\dagger} \hat{b}\right)$, which in the case of $\omega_{F}=\omega_{A}$ has the re- lation of $\left[H_{F A}, H\right]=\omega^{\prime}\left(\omega_{F}-\omega_{A}\right)\left(\hat{a} \hat{b}^{\dagger}-\hat{a}^{\dagger} \hat{b}\right)=0$. After some simple algebra, we obtain (for $\omega_{A}=\omega_{F}$ )

$$
d\left\langle H_{F A}\right\rangle / d t=-\gamma_{F}\left\langle H_{F A}\right\rangle / 2
$$

or

$$
d\left\langle H_{F A}\right\rangle / d t=-\left(n_{A}+1 / 2\right) \gamma_{F}\left\langle H_{F A}\right\rangle
$$

for the $T_{1}$ relaxation via the dissipative mode $W_{F}=\hat{a}$ or $W_{A}=\hat{b}$, respectively. In either case, we have that in the conventional CQED formulation, $\left\langle H_{F A}\right\rangle=0$ if it was initially zero. Therefore, the nonzero $\left\langle H_{F A}\right\rangle$ may be used as a sensitive indicator for the breakdown of the conventional theory.
[1] U. Weiss, Quantum Dissipative Systems, 2nd ed., Series in Modern Condensed Matter Physics Vol. 10 (World Scientific, Singapore, 1999).

[2] H. Risken, The Fokker-Planck Equation, 2nd ed. (Springer, Berlin, 1984).

[3] H. Mori, Prog. Theor. Phys. 33, 423 (1965).

[4] R. W. Zwanzig, J. Chem. Phys. 33, 1338 (1960).

[5] S. Nakajima, Prog. Theor. Phys. 20, 948 (1958).

[6] R. P. Feynman and J. F. L. Vernon, Ann. Phys. (N.Y.) 24, 118 (1963).

[7] A. O. Caldeira and A. J. Leggett, Physica A 121, 587 (1983).

[8] A. J. Leggett, S. Chakravarty, A. T. Dorsey, and M. Gary, Rev. Mod. Phys. 59, 1 (1987).

[9] H. Haken, Rev. Mod. Phys. 47, 67 (1975).

[10] W. H. Louisell, Quantum Statistical Properties of Radiation (Wiley, New York, 1973).

[11] Y. J. Yan and S. Mukamel, J. Chem. Phys. 89, 5160 (1988).

[12] G. Lindblad, Commun. Math. Phys. 48, 119 (1976).

[13] V. Gorini, A. Kossakowski, and E. C. G. Sudarshan, J. Math. Phys. 17, 821 (1976).

[14] R. Alicki and K. Lendi, in Quantum Dynamical Semigroups and Applications, Lecture Notes in Physics Vol. 286 (Springer, New York, 1987).

[15] Y. Tanimura and P. G. Wolynes, Phys. Rev. A 43, 4131 (1991).

[16] Y. Tanimura and P. G. Wolynes, J. Chem. Phys. 96, 8485 (1992).

[17] Y. Tanimura and Y. Maruyama, J. Chem. Phys. 107, 1779 (1997).

[18] C. Meier and D. J. Tannor, J. Chem. Phys. 111, 3365 (1999).

[19] Y. J. Yan, Phys. Rev. A 58, 2721 (1998).

[20] R. X. Xu and Y. J. Yan (unpublished).

[21] Y. J. Yan, F. Shuang, R. X. Xu, J. X. Cheng, X. Q. Li, C. Yang, and H. Y. Zhang, J. Chem. Phys. 113, 2068 (2000).

[22] R. K. Wangsness and F. Bloch, Phys. Rev. 89, 728 (1953).

[23] F. Bloch, Phys. Rev. 105, 1206 (1957).

[24] A. G. Redfield, Adv. Magn. Reson. 1, 1 (1965).

[25] F. Shuang, C. Yang, and Y. J. Yan, J. Chem. Phys. 114, 3868 (2000)
[26] J. Rarity and C. Weisbuch, Microcavities and Photonic Bandgaps: Physics and Applications (Kluwer, Dordrecht, 1996).

[27] A. Barenco, D. Deutsch, A. Ekert, and R. Jozsa, Phys. Rev. Lett. 74, 4083 (1995).

[28] T. Sleator and H. Weinfurter, Phys. Rev. Lett. 74, 4087 (1995).

[29] P. Domokos, J. M. Raimond, M. Brune, and S. Haroche, Phys. Rev. A 52, 3554 (1995).

[30] Q. A. Turchette, C. J. Hood, W. Lange, H. Mabuchi, and H. J. Kimble, Phys. Rev. Lett. 75, 4710 (1995).

[31] A. Rauschenbeutel, G. Nogues, S. Osnaghi, P. Bertet, M. Brune, J. M. Raimond, and S. Haroche, Phys. Rev. Lett. 83, 5166 (1999).

[32] M. S. Sherwin, A. Imamoglu, and T. Montroy, Phys. Rev. A 60, 3508 (1999).

[33] A. Imamoglu, D. D. Awschalom, G. Burkard, D. P. DiVincenzo, D. Loss, M. Sherwin, and A. Small, Phys. Rev. Lett. 83, 4204 (1999).

[34] S. Haroche and D. Kleppner, Phys. Today 42(1), 24 (1989).

[35] P. R. Berman, Cavity Quantum Electrodynamics (Academic, New York, 1995).

[36] E. Burstein and C. Weisbush, Confined Electrons and Photons (Plenum, New York, 1995).

[37] E. Yablonovitch, T. J. Gmitter, and R. Bhat, Phys. Rev. Lett. 61, 2546 (1988).

[38] H. Yokoyama, K. Nishi, T. Anan, H. Yamada, S. D. Brorson, and E. P. Ippen, Appl. Phys. Lett. 57, 2814 (1990).

[39] G. Björk, S. Machida, Y. Yamamoto, and K. Igeta, Phys. Rev. A 44, 669 (1991).

[40] C. Weisbuch, M. Nishioka, A. Ishikawa, and Y. Arakawa, Phys. Rev. Lett. 69, 3314 (1992).

[41] E. T. Jaynes and F. W. Cummings, Proc. IEEE 51, 89 (1963).

[42] J. D. Cresser, J. Mod. Opt. 11, 2187 (1992).

[43] L. Gammaitoni, P. Hänggi, P. Jung, and F. Marchesoni, Rev. Mod. Phys. 70, 223 (1998).

[44] D. E. Makarov and N. Makri, Phys. Rev. B 52, R2257 (1995).

[45] F. Shuang, C. Yang, H. Zhang, and Y. J. Yan, Phys. Rev. E 61, 7192 (2000). 